GPS $97 / 11$

April 1997

\title{
Open supermembranes in eleven dimensions
}

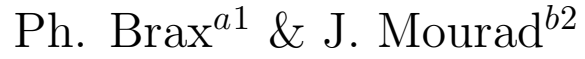 \\ a Service de Physique Théorique, CEA-Saclay \\ F-91191 Gif/Yvette Cedex, France \\ ${ }^{b}$ GPS, Université de Cergy-Pontoise \\ Site Saint-Martin, F-95302 Cergy-Pontoise, France
}

\begin{abstract}
We consider open supermembranes in an eleven dimensional background. We show that, in a flat space-time, the world-volume action is kappa-symmetric and has global space-time supersymmetry if space-time has even dimensional topological defects where the membrane can end. An example of such topological defects is provided by the space-time with boundaries considered by Horava and Witten. In that case the world-volume action has reparametrisation anomalies whose cancellation requires the inclusion of a current algebra on the boundaries of the membrane. The role of kappa-anomalies in a general background is discussed. The tension of the membrane is related to the eleven dimensional gravitational constant with the aid of the Green-Schwarz mechanism allowing a consistency check of $M$-theory.
\end{abstract}

\footnotetext{
${ }^{1}$ email: brax@spht.saclay.cea.fr

2email:mourad@qcd.th.u-psud.fr
} 


\section{Introduction}

One of the most fascinating aspects of recent progress towards a non-perturbative understanding of string theory is the role played by an eleven dimensional theory, $M$-theory, whose low energy limit is given by the eleven dimensional supergravity. In particular, type IIA superstring is believed to be related to $M$-theory compactified on a circle [1, 2] and there are many indications that the $E_{8} \times E_{8}$ heterotic string is related to $M$-theory compactified on an interval $\left(S^{1} / Z_{2}\right)$ [3, , 4]. The relation of type IIA superstrings to $M$-theory is supported by the existence of a closed supermembrane in eleven dimensions [5, 6] whose double dimensional reduction, that is the simultaneous reduction of both a space-time and a worldvolume coordinate, leads to a type IIA superstring [7]. The role of the closed supermembrane in eleven dimension was strengthened in [5] where it was shown that kappa-symmetry of the worldvolume action leads to the 11D SUGRA equations of motion. The identification of the eleven-dimensional Kaluza-Klein states with type IIA D-0 branes [2, 8, 9] put the conjecture that the strong coupling limit of IIA superstring is eleven dimensional supergravity on a firm basis. On the other hand, the strong coupling limit of the $E_{8} \times E_{8}$ heterotic string is believed to be given by the eleven-dimensional supergravity with one dimension being an interval, the $E_{8}$ gauge fields and their superpartners living on the boundaries of space-time [3, 4]. The arguments given by Horava and Witten in favor of this conjecture include the consideration of the degrees of freedom of the solitonic membrane and especially the cancellation of space-time gravitational anomalies [3]. The anomaly cancellation argument was developed further in [4] (see also [10, 11]); together with the requirement of local supersymmetry it allowed to determine perturbatively the Lagrangian describing the low energy strong coupling limit of the $E_{8} \times E_{8}$ heterotic string [4].

The aim of this paper is to examine further the membrane arguments in order to have explicit relations with the heterotic string at the world-volume level, the heterotic string being obtained by a double dimensional reduction of the open supermembrane in the same manner that type IIA superstring is obtained by a double dimensional reduction of the closed supermembrane. Open supermembranes were considered in [6] in an eleven dimensional background with no topological defects. It was noticed there that global supersymmetry cannot be achieved. We return to this issue in section 2 where we prove that global supersymmetry and kappa-symmetry can be achieved when space-time has topological defects where the membrane can end. These topological defects are the analogue of D-branes for type II superstrings. One of these topological defects consists of having a spacetime with boundaries in the manner considered by Horava and Witten. We also examine the boundary conditions in this section. In section 3 we consider worldvolume reparametrisation anomalies and add to the action a suitable boundary term in order to cancel anomalies. We discuss the different cases corresponding to the membrane having its two ends on one boundary of space-time or on the 
two boundaries. We also discuss the effect of a topological three dimensional term which moves the anomaly from one boundary to the other. In section 4 we sketch the consequences of coupling the supermembrane to background fields, we emphasize the role of kappa-anomalies in deriving the classical equations of motion and especially in giving the boundary value of the four-form field strength. We show how kappa-anomalies rule out all the configurations of the supermembrane except the one corresponding to having one $E_{8}$ on each boundary of space-time. In section 5 we show how the eleven-dimensional Green-Schwarz mechanism [4] allows the prediction of the eleven-dimensional gravitational constant in terms of the membrane tension. We collect our conclusions in section 6 .

\section{The action and its symmetries}

We first consider a flat background. We denote the embedding of the supermembrane in eleven dimensional superspace by $Z^{M}\left(\xi^{i}\right)=\left(X^{a}\left(\xi^{i}\right), \theta^{\alpha}\left(\xi^{i}\right)\right), a=$ $1, \ldots 11, \alpha=1 \ldots 32, i=1,2,3$. The moving basis is $E^{a}=d X^{a}-i \overline{d \theta} \Gamma^{a} \theta, E^{\alpha}=$ $d \theta^{\alpha}$. The action of the open supermembrane, given in the Dirac-Nambu-Goto form, is

$$
S=-T_{3}\left[\int_{\Sigma_{3}} \sqrt{-\tilde{g}}+\int_{\Sigma_{3}} \tilde{C}+\int_{\partial \Sigma_{3}} \tilde{B}\right]
$$

where $\tilde{g}_{i j}$ is the induced metric

$$
\tilde{g}_{i j}=\tilde{E}_{i}^{a} \tilde{E}_{j}^{b} \eta_{a b}
$$

$\tilde{E}_{i}^{a} d \xi^{i}$ are the pullback of the local frame, $\tilde{C}$ is the pullback of the eleven dimensional super three-form and $\tilde{B}$ is the pullback of a super two-form potential into the world-volume 3 . The last term in the action, which is absent for a closed supermembrane, is added in order to respect the gauge invariance:

$$
C \rightarrow C+d \Lambda
$$

where $\Lambda$ is a two-form. The action is invariant under this transformation if the two-form potential transforms as

$$
B \rightarrow B-\Lambda
$$

Eleven-dimensional supergravity has no two-form potential in its spectrum. this is the first indication that in order to have open supermembranes one needs some topological defects localised in a supersubmanifold $\mathcal{M}^{\prime}$ where this twoform potential can live. The boundary of the supermembrane must lie on these topological defects.

\footnotetext{
${ }^{3}$ In the following we will denote the pullback into the world-volume of a space-time form $A$ by $\tilde{A}$.
} 
An essential requirement for an acceptable action is its kappa-symmetry. The kappa-transformation of the pullback of a space-time form $A$ is conveniently given by

$$
\delta_{\kappa} \tilde{A}=\widetilde{L_{\kappa} A},
$$

where $L_{\kappa}=d \iota_{\kappa}+\iota_{\kappa} d$ is the Lie derivative with respect to the vector field $\kappa$ :

$$
\kappa=\Delta^{\alpha}(Z) E_{\alpha}
$$

$\Delta$ being local fermionic parameters constrained by

$$
\tilde{\Delta}=\tilde{\Gamma} \tilde{\Delta}
$$

where $\tilde{\Gamma}$ is given by

$$
{ }^{*} \tilde{\Gamma}=\frac{1}{3 !} E^{a} E^{b} E^{c} \Gamma_{a b c},
$$

the $*$ represents the hodge dual on the world-volume. The kappa variation of the induced volume element is thus given by

$$
\delta_{\kappa} \sqrt{-\tilde{g}}=\frac{1}{2} \sqrt{-\tilde{g}} \tilde{g}^{i j} \delta_{\kappa} \tilde{g}_{i j}=-i \epsilon^{i j k}\left(\gamma_{j k}\right)_{\beta \alpha} \tilde{\Delta}^{\alpha} \tilde{E}_{i}^{\beta}
$$

where use has been made of the constraint (7), the definition $\gamma_{i} d \xi^{i}=\widetilde{\Gamma_{a} E^{a}}$, as well as the identity

$$
\sqrt{-\tilde{g}} \tilde{g}^{i j} \gamma_{j} \tilde{\Gamma}=\epsilon^{i j k} \gamma_{j k}
$$

Finally the kappa-variation of the action can be written as

$$
\delta_{\kappa} S=-T_{3}\left[\int_{\Sigma_{3}}\left(-i \epsilon^{i j k}\left(\gamma_{j k}\right)_{\beta \alpha} \tilde{\Delta}^{\alpha} \tilde{E}_{i}^{\beta}+\widetilde{\iota_{\kappa} G}\right)+\int_{\partial \Sigma_{3}} \widetilde{\iota_{\kappa} H}\right]
$$

where we defined the field strengths

$$
G=d C, \quad H=d B+C .
$$

Note that $G$ and $H$ are invariant under the gauge transformations (3, (1). The conditions for kappa-symmetry are thus

$$
\begin{array}{r}
G=i\left(\Gamma_{a b}\right)_{\alpha \beta} E^{\alpha} E^{\beta} E^{a} E^{b}+G^{\prime}, \quad \iota_{E_{\alpha}} G^{\prime}=0 \\
\iota_{E_{\alpha}} H=0, \alpha=1, \ldots 32 .
\end{array}
$$

The first term in $G$ is closed due to a gamma matrix identity in eleven dimensions [5]. The variation of the action under a global super Poincaré transformation generated by the vector field $P$ is given by

$$
\delta_{P} S=-T_{3}\left[\int_{\Sigma_{3}} \widetilde{\iota_{P} G}+\int_{\partial \Sigma_{3}} \widetilde{\iota_{P} H}\right]
$$


The action is thus invariant under the transformations satisfying

$$
L_{P} G=0, \quad L_{P} H=0 .
$$

The combination of kappa-symmetry and global super Poincaré symmetry imposes the relations

$$
G=i\left(\Gamma_{a b}\right)_{\alpha \beta} E^{\alpha} E^{\beta} E^{a} E^{b}, \quad H=0 .
$$

The latter relation being verified on a supersubmanifold, $\mathcal{M}^{\prime}$, of the eleven dimensional super space-time where the boundary of the membrane lies. The field strengths $G$ and $H$ are not independent, they are related by

$$
d H=\left.G\right|_{\mathcal{M}^{\prime}},
$$

so we get the important constraint

$$
\left.G\right|_{\mathcal{M}^{\prime}}=0 .
$$

Before analysing equation (19) we examine the boundary conditions which are compatible with the requirement

$$
\partial \Sigma_{3} \subset \mathcal{M}^{\prime} .
$$

Boundary conditions are obtained by considering an arbitrary variation of the action in order to obtain the equations of motion and demanding that the boundary terms vanish. Let the even part of $\mathcal{M}^{\prime}$ be described by

$$
X^{\bar{a}}=0, \bar{a}=11-d+1, \ldots, 11,
$$

where $d$ is the dimension of $\mathcal{M}^{\prime}$, use the splitting $X^{a}=\left(X^{\underline{a}}, X^{\bar{a}}\right)$, and suppose that the boundary of the membrane is given by $\xi^{3}=0$ then the boundary conditions compatible with (20) are

$$
\left.X^{\bar{a}}\right|_{\partial \Sigma_{3}}=0,\left.\quad \tilde{E}_{3}^{a}\right|_{\partial \Sigma_{3}}=0 .
$$

Supersubmanifolds $\mathcal{M}^{\prime}$ where (19) holds are given by

$$
d=2 n, \quad \theta=\Gamma^{1} \Gamma^{2} \ldots \Gamma^{d} \theta, n=0, \ldots 5 .
$$

These solutions preserve the supersymmetries that obey

$$
\epsilon=\Gamma^{1} \Gamma^{2} \ldots \Gamma^{d} \epsilon .
$$

The case with $d=10$ represents the Horava-Witten boundary of space-time, the case with $d=6$ is the M-theory fivebrane. The interpretation of the elevendimensional fivebrane as a Dirichlet Brane for membranes was proposed previously in [12]. The possibility that membranes can end on fivebranes was pointed 
out in [13] using charge conservation arguments. The existence of ninebranes

$(d=10)$ in eleven dimensions was conjectured in [14, 15, 16]. Twisted membranes with a action different from ours were considered in [17]. The list given in (23) includes four new branes in eleven dimensions $(\mathrm{p}=-1,1,3,7)$. The existence of these objects in eleven dimensions needs further support. Here we simply note that these objects allow the open supermembrane to end while preserving half of the eleven dimensional supersymmetries, whether they all exist is beyond the scope of the present article. In the rest of the paper we will focus our attention on the $d=10$ case which we consider as the boundary of space-time.

\section{Reparametrisation anomalies}

Let the eleven dimensional space-time have the topology $R^{10} \times[0, l]$, with the boundaries located at $x^{11}=0$ and $x^{11}=l$. The boundaries of the open supermembrane must, according to the preceding section, lie on $x^{11}=0$ and/or $x^{11}=l$. The Horava-Witten configuration, where on each boundary lives an $E_{8}$ supermultiplet, corresponds, as we will see, to one boundary of the supermembrane on $x^{11}=0$ and the other boundary on $x^{11}=l$. The topology of the membrane that we shall consider is $\Sigma_{2} \times I$ with $\Sigma_{2}$ a closed two-dimensional surface, and $I=[0, \pi]$ an interval with coordinate $\xi^{3}$. The case corresponding to the Horava-Witten configuration is obtained by setting $\xi^{3}=\pi x^{11} / l$, while the case where the two ends of the membrane lie on $x^{11}=0$ is obtained by setting, e.g., $x^{11}=l^{\prime} \sin \left(\xi^{3}\right)$, with $l^{\prime}<l$.

The action (11), with these conditions, reads, in the Polyakov form,

$$
S=-T_{3}\left[\frac{1}{2} \int_{\Sigma_{3}}\left(\sqrt{-g}\left(g^{i j} \tilde{g}_{i j}-1\right)+\tilde{C}\right)+\int_{\Sigma_{2}, \xi^{3}=\pi} \tilde{B}-\int_{\Sigma_{2}, \xi^{3}=0} \tilde{B}\right]
$$

where we introduced the word-volume metric $g_{i j}$ as an auxiliary field. The worldvolume fields of the supermembrane can be developed in Kaluza-Klein modes in the $\xi^{3}$ direction. The potential $B$ is defined only on the boundary and thus has no $\xi^{3}$ expansion. The resulting zero-modes coincide with that of the supercoordinates of the GS formulation of the heterotic string in ten dimensions with $\alpha^{\prime}$ given by

$$
l T_{3}=\frac{1}{2 \pi \alpha^{\prime}}
$$

and a WZW term given by

$$
\frac{1}{2 \pi \alpha^{\prime}} \int_{\Sigma_{2}} \tilde{B}^{\prime}
$$

with $B^{\prime}$ a super two-form given by

$$
B^{\prime}=\frac{1}{l}\left(B\left(x^{11}=l\right)-B\left(x^{11}=0\right)\right)+\iota_{E^{11}} C
$$


for the Horava-Witten case and

$$
2 l^{\prime} T_{3}=\frac{1}{2 \pi \alpha^{\prime}}, \quad B^{\prime}=\iota_{E^{11}} C
$$

for the case where the two ends of the membrane are on one boundary. Note that the effective two-form (28) obtained by a dimensional reduction of the membrane, in the Horava-Witten configuration, involves the two different two-forms on the boundaries of space-time as well as the reduction of the three form potential.

The resulting zero-mode action has world-sheet reparametrisation anomalies which were calculated in [18] and shown to be given by

$$
X_{4}=d X_{3}=-\frac{1}{6 \pi} \operatorname{tr}\left(\mathcal{R}^{2}\right)
$$

where $\mathcal{R}$ is the two-dimensional curvature. A three dimensional theory on a smooth manifold has no anomalies [19], so, as in the Horava-Witten construction, the anomalies must be concentrated on the boundaries of the membrane. One expects the anomalies to be distributed symmetrically between the two boundaries, that is each boundary supports half of the total anomaly

$$
\delta \Gamma=\frac{1}{2} \int_{\Sigma_{2}, \xi^{3}=\pi} X_{2}^{1}+\frac{1}{2} \int_{\Sigma_{2}, \xi^{3}=0} X_{2}^{1},
$$

where $\delta X_{3}=d X_{2}^{1}$. Note however that it is possible to add to the world-volume action the term

$$
-\frac{1}{2} \int_{\Sigma_{3}} X_{3}
$$

whose effect is to remove the anomaly from one side to add it on the other side. In fact the variation under reparametrisation of the term (32) can be written as

$$
-\frac{1}{2} \int_{\Sigma_{3}} d X_{2}^{1}=\frac{1}{2} \int_{\Sigma_{2}, \xi^{3}=0} X_{2}^{1}-\frac{1}{2} \int_{\Sigma_{2}, \xi^{3}=\pi} X_{2}^{1}
$$

A counter term analogous to (32) is also possible in the eleven-dimensional analysis of the gravitational anomaly, namely the addition to the eleven-dimensional supergravity action of $\int_{M_{11}} I_{11}$, where $I_{12}=d I_{11}$ represents the ten dimensional gravitational anomaly. The $E_{8} \times E_{8}$ heterotic string, with each $E_{8}$ propagating on one boundary, was related in [3, 4] to the case were the anomaly is symmetrically distributed between the two boundaries of space-time. The inclusion of the topological term $\int I_{11}$ allows to argue for the possibility of having $E_{8} \times E_{8}$ or a $S O(32)$ on one boundary and no matter on the other boundary. This picture is compatible, as we will see, with the world-volume reparametrisation anomaly cancellation but needs further analysis on the eleven-dimensional level where the Green-Schwarz mechanism is not expected to arise in a natural way from the Chern-Simons terms of eleven-dimensional supergravity as is the case for the Horava-Witten case. We shall return to this point in the next sections. 


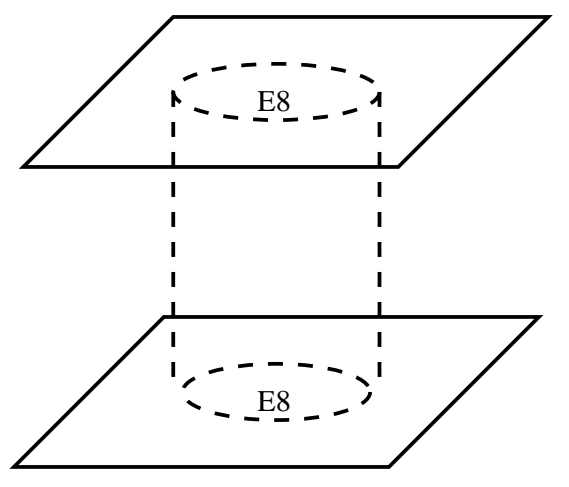

A
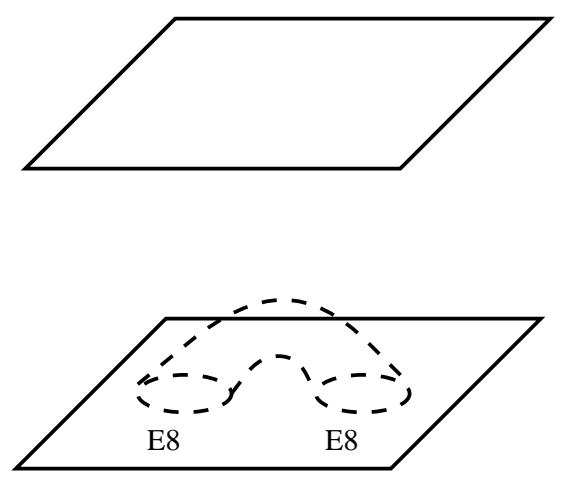

C

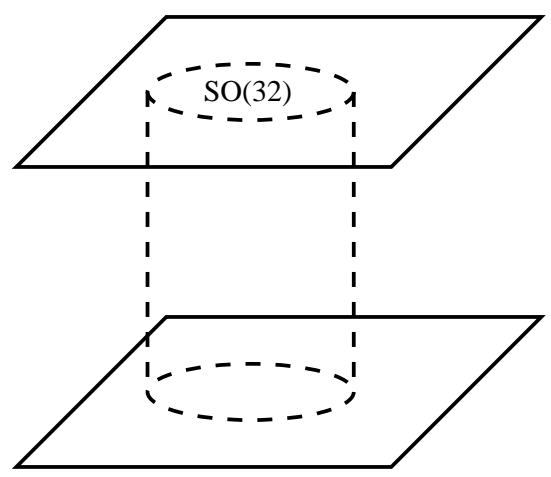

B
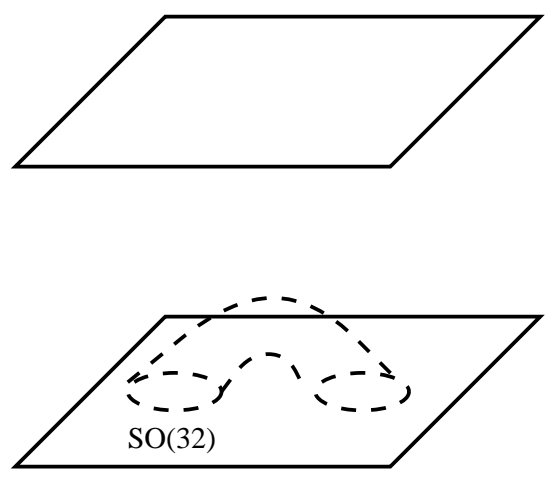

D

Fig. : Different configurations of the membrane.

The current algebras on each boundary are specified. 
Suppose first that the anomaly on the world-volume is distributed symmetrically between the two boundaries, then one has to add 16 two-dimensional Majorana-Weyl fermions on each boundary. The 16 fermions form an $E_{8}$ current algebra. We can have two situations: i) the two boundaries of the membrane are on $x^{11}=0$, then one has an $E_{8} \times E_{8}$ gauge multiplet on $x^{11}=0$ (fig. $\mathrm{C}$ ); ii) one boundary of the membrane is on $x^{11}=0$ and the other is on $x^{11}=l$, then one has one $E_{8}$ propagating on each boundary and recovers the Horava-Witten construction (fig. A).

Suppose next that, with the aid of the three dimensional topological term, all the anomaly is concentrated on one boundary, then one has to add on this boundary 32 Majorana-Weyl fermions. They form the current algebra of $S O(32)$ or $E_{8} \times E_{8}$. This case, wherever are the two boundaries of the membrane (fig.B, D), corresponds to a $S O(32)$ or $E_{8} \times E_{8}$ multiplet propagating on one boundary of space-time.

The boundary term required for the anomaly cancellation which must be added to the action (25) (and depending on the situation considered to (32) ) is of the form

$$
\frac{1}{4 \pi \alpha^{\prime}} \int_{\Sigma_{2}} \sqrt{-g} \psi^{t} \partial_{-} \psi
$$

where $g$ is the restriction of the three dimensional metric on the boundary and $\psi$ represents a multiplet of 16 or 32 world-sheet Majorana-Weyl fermions. These fermions, in flat space-time, must be invariant under kappa transformations and global supersymmetry.

In brief, we found in this section that the cancellation of world-volume reparametrisation anomalies leads to five different configurations depending on whether the membrane starts and ends on the same boundary and whether the current algebra is on either the same boundary or on both. In the next section all of these configurations except the Horava-Witten case will be ruled out by the requirement of one-loop kappa-anomaly cancellation in a general background.

\section{General background and kappa anomalies}

The generalisation of the preceding action to curved space-time with a coupling to a super Yang-Mills potential $A$ on the boundary is straightforward. The bulk action is analogous to the closed membrane action considered in [5] and the fermion boundary term is analogous to the one existing in the heterotic string [20]. The classical kappa-symmetry of the action imposes constraints on the background superfields which are, after field redefinitions, those of the superfield formulation of eleven-dimensional supergravity [5] and supersymmetric YangMills in ten dimensions [20] with the boundary condition

$$
d H=\left.G\right|_{\mathcal{M}^{\prime}}=0
$$


As explained in 21] for the ten dimensional heterotic string, the coupling between the two sectors arises when one considers quantum kappa-anomalies. As noted in the preceding section, the zero-modes of the action is the GS formulation of the heterotic string so the action is kappa-anomalous with the anomalies concentrated on the boundaries of the membrane. The one loop total anomaly found in [22] may be written as

$$
\delta_{\kappa} \Gamma=-\frac{1}{8 \pi} \int_{\Sigma_{2}} \widetilde{\iota_{\kappa} \omega}
$$

with the the three- superform $\omega$ given by

$$
\omega=\omega_{3 Y M}-\omega_{3 L}
$$

$\omega_{3 Y M}$ and $\omega_{3 L}$ being the Yang-Mills and Lorentz Chern-Simons forms, so that

$$
d \omega=\operatorname{tr}\left(F^{2}\right)-\operatorname{tr}\left(R^{2}\right)
$$

Let us first consider the case where one boundary of the membrane is on $x^{11}=0$ and the other on $x^{11}=l$ and where there is on each boundary an $E_{8}$ current algebra (fig. A). This case is characterized, as we have seen, by a symmetrical distribution of the world-sheet reparametrisation and space-time gravitational anomalies. The kappa-anomaly in this case reads

$$
\delta_{\kappa} \Gamma=-\frac{1}{8 \pi}\left[\int_{\Sigma_{2}, \xi^{3}=\pi} \widetilde{\iota_{\kappa} \omega_{1}}+\int_{\Sigma_{2}, \xi^{3}=0} \widetilde{\iota_{\kappa} \omega_{2}}\right]
$$

with $\omega_{i}=\omega_{3 Y M}{ }_{i}-\frac{1}{2} \omega_{3 L}$ the index $i$ representing the two different $E_{8}$ gauge fields. The structure of these anomalies is similar to the last term in (11). This suggests that their cancellation can be achieved by the replacement of $H$ by $H^{\prime}$ with

$$
\begin{gathered}
H^{\prime}=H+\frac{1}{8 \pi T_{3}} \omega_{1}, \quad \text { at } x^{11}=l \\
H^{\prime}=H-\frac{1}{8 \pi T_{3}} \omega_{2}, \quad \text { at } x^{11}=0 .
\end{gathered}
$$

Using the fact the $d H^{\prime}=0$ we get

$$
\left.G\right|_{\mathcal{M}^{\prime}, x^{11}=l}=\frac{1}{8 \pi T_{3}}\left(\operatorname{tr}\left(F_{1}^{2}\right)-\frac{1}{2} \operatorname{tr}\left(R^{2}\right)\right) \equiv-\frac{1}{8 \pi T_{3}} I_{4}
$$

and an analogous result with the opposite sign at $x^{11}=0$ which replaces the boundary condition (35). This constitutes an alternative derivation of the result of Horava and Witten where the value of $G$ at the boundary was fixed by the requirement of local supersymmetry and gravitational anomaly cancellation. Our relation is expressed in terms of the membrane tension whereas the relation of 
[4] is expressed in terms of the gauge and coupling constant. This will allow, in the next section, a consistency check of $M$ theory.

The other configurations can be studied in a similar way. The above mechanism for the cancellation of kappa-anomalies is not possible when the membrane starts and ends on the same boundary (figs. C,D) because the the $H$ cannot de modified in two different ways on the same space-time boundary.

Consider next the $S O(32)$ (or $E_{8} \times E_{8}$ ) case where the gauge supermultiplet is on $x^{11}=l$ and the boundary of the membrane carrying the $S O(32)$ (or $E_{8} \times E_{8}$ ) current algebra is on $x^{11}=l$ (fig. B). The one loop kappa-anomaly in this case is given by

$$
\delta_{\kappa} \Gamma=-\frac{1}{8 \pi}\left[\int_{\Sigma_{2}, \xi^{3}=\pi} \widetilde{\iota_{\kappa} \omega^{\prime}}+\int_{\Sigma_{2}, \xi^{3}=0} \widetilde{\iota_{\kappa} \omega^{\prime \prime}}\right]+\frac{1}{2} \int_{\Sigma_{3}} \delta_{\kappa} X_{3},
$$

where $\omega^{\prime}=\omega_{3 Y M}-\frac{1}{2} \omega_{3 L}$ and $\omega^{\prime \prime}=-\frac{1}{2} \omega_{3 L}$. The fact that all the anomaly originating from the gauge background is located at $x^{11}=l$ is due to the fact that the gauge part of the anomaly is due to the heterotic fermions. The precise value of $\delta_{\kappa} X_{3} \neq 0$ is not relevant. The fact that the anomaly mixes terms which are pullback fields and world-volume fields makes it impossible to modify $H$ in such a way as to cancel the anomaly. So we conclude that this case is also ruled out since it is kappa-anomalous.

In brief, only the configuration cooresponding to the Horava-Witten case (fig. (A)) is non-anomalous. At the eleven-dimensional level, the same result has an apparently different origin. In fact the anomaly cancellation in eleven dimensions is due to the presence in the Lagrangian of two topological terms, the first is the Chern-Simons interaction $\int C G^{2}$ and the second is of the form $\int C X_{8}$ with $X_{8}$ a quartic polynomial in the curvature given by

$$
X_{8}=-\frac{1}{8} \operatorname{tr}\left(R^{4}\right)+\frac{1}{32}\left(\operatorname{tr}\left(R^{2}\right)\right)^{2} ;
$$

the latter term can de deduced from anomaly cancellation argument in the fivebrane [23, 24] or from the type IIA superstring after a compactification on $S^{1}$ [25]. For the Green-Schwarz mechanism to work, with no other terms added to the Lagrangian, the anomaly, on each boundary, must be factorizable as $I_{12} \propto$ $I_{4}\left(I_{4}^{2} / 4-X_{8}\right)$ where $I_{4}$ is proportional to the value of $G$ at the boundary. It turns out that this is only true for the Horava-Witten configuration.

\section{The Green-Schwarz mechanism in eleven di- mensions}

In the preceding section it was shown that $H$ must be modified in order to cancel kappa-anomalies as

$$
H^{\prime}=C+d B+\frac{1}{8 \pi T_{3}} \omega_{1}
$$


on $x^{11}=l$. Under a gauge or Lorentz transformation $H^{\prime}$ must be invariant, this fixes the variation of $C+d B$ as

$$
\delta(C+d B)=-\frac{1}{8 \pi T_{3}} d \omega_{2}^{1}
$$

where $\delta \omega_{1}=d \omega_{2}^{1}$. By a gauge transformation (3, 4) it is possible to choose

$$
\delta C=0, \quad \delta B=-\frac{1}{8 \pi T_{3}} \omega_{2}^{1}
$$

The resulting transformation of the effective two-form potential $B^{\prime}$ (28) is thus given by

$$
\delta B^{\prime}=-\frac{\alpha^{\prime}}{4}\left(\omega_{2 Y M 1}^{1}+\omega_{2 Y M 2}^{1}-\omega_{2 L}^{1}\right),
$$

which is the usual transformation of the two-form potential in ten dimensional string theory. The transformations (47) raise the problem of understanding how the Green-Schwarz mechanism can cancel the anomalies in eleven dimensions since $C$ is invariant. The solution to this problem consists in first noticing that the topological terms in the low energy limit of $M$-theory f,

$$
S_{T}=-\frac{1}{6 k_{11}^{2}} \int_{M_{11}} C \wedge G^{2}+\frac{T_{3}}{12(2 \pi)^{4}} \int_{M_{11}} C \wedge X_{8}
$$

are not invariant under the gauge tranformation (3, 4) [11]; so in order to restore this gauge invariance one has to add to the action, the boundary terms

$$
\Delta S_{T}=-\frac{1}{6 k_{11}^{2}} \int_{\partial M_{11}} B \wedge G^{2}+\frac{T_{3}}{12(2 \pi)^{4}} \int_{\partial M_{11}} B \wedge X_{8}
$$

The gauge where (47) is true is natural because the anomaly is concentrated at the boundary and its cancellation is realised by fields which live on the boundary. The gauge chosen in 4 , 10] corresponds to setting $\delta B=0$ and this requires to have a variation of $C$ which is non-zero in the bulk [11]. Recall that the anomaly at $x^{11}=l$ is given by

$$
\delta \Gamma=-\frac{1}{48(2 \pi)^{5}} \int \omega_{2}^{1} \wedge\left(\frac{I_{4}^{2}}{4}-X_{8}\right) .
$$

On the other hand, the variation of the ten dimensional topological terms is given by

$$
\delta \Delta S_{T}=\frac{1}{6 k_{11}^{2}}\left(\frac{1}{8 \pi T_{3}}\right)^{3} \int \omega_{2}^{1} \wedge I_{4}^{2}-\frac{1}{48(2 \pi)^{5}} \int \omega_{2}^{1} \wedge X_{8} .
$$

\footnotetext{
${ }^{4}$ Our conventions are those of the second reference in [10] they are related to those of [4] by $C=6 \sqrt{2} C^{H W}, G=\sqrt{2} G^{H W}$ and to those of [23] by $k_{11}^{2}=2 k_{11}^{2 D L M}$.
} 
Comparaison with (51) shows that the $X_{8}$ part of the anomaly cancels and that the remaing part is cancelled if the membrane tension and the gravitational constant are related by

$$
k_{11}^{2}=\frac{2 \pi^{2}}{T_{3}^{3}} .
$$

A relation similar to (53) was derived in different manners: in [23] (see also [10, 26]) it was derived with the aid of the quantization of the flux of $G$, in [10] it was derived using the D-brane tension formulae given in [8]. Here we have given yet another derivation combining the world-volume and space-time anomaly cancellation. This is an additional indication of the important role that open supermembranes have in $M$-theory.

Before ending this section let us note that the gauge coupling constant can also be determined in this framework. In fact the value of $G$ at the boundary determined in [4] reads, in our notations,

$$
\left.G\right|_{x^{11}=l}=-\frac{k_{11}^{2}}{2 \lambda^{2}} I_{4},
$$

where $\lambda$ is the gauge coupling constant. Comparing this relation with our relation (42), we get

$$
\lambda^{2}=4 \pi T_{3} k_{11}^{2}=8 \frac{\pi^{3}}{T_{3}^{2}}=2 \pi\left(4 \pi k_{11}^{2}\right)^{2 / 3} .
$$

The last expression is the one derived in [4].

\section{Conclusion}

We showed that open supermembranes propagate in eleven dimensions provided the boundaries of the membrane lie on an even dimensional submanifold, the action having half the supersymmetries of eleven-dimensions. We studied the open membrane in the space-time considered by Horava and Witten. The cancellation of world-volume reparametrisation anomalies allows many configurations depending on whether the membrane starts and ends on either the same spacetime boundary or on both; and on how to distribute the anomaly between the two boundaries of the membrane. We showed that all these configurations, except the case where each boundary carries an $E_{8}$ current algebra and lies on a different space-time boundary, are kappa-anomalous. This provides a further confirmation, at the supermembrane level, of the result of Horava and Witten that the only possibility on $M_{10} \times S^{1} / Z_{2}$ is $E_{8} \times E_{8}$ with each factor propagating on a boundary. In addition it gives an alternative derivation of the value at the boundary of the four-form field strength and the relation between the membrane tension and the gravitational constant. This relation is compatible with other independent derivations and thus constitutes a positive consistency check of $M$-theory. 
A similar study for membranes in an eleven-dimensional background with five-branes where the membrane can have its boundaries would be of interest, especially that the five-brane action is now known [27, 28].

Acknowledgements: We would like to thank E. Dudas for many helpful remarks.

\section{References}

[1] P.K. Townsend, The 11-dimensional supermembrane revisited, Phys. Lett. B350 (1995) 184.

[2] E. Witten, String theory dynamics in various dimensions, Nucl. Phys. B443 (1995) 85.

[3] P. Horava and E. Witten, Heterotic and Type I string dynamics from eleven dimensions, Nucl. Phys. B460 (1996) 506.

[4] P. Horava and E. Witten, Eleven-dimensional supergravity on a manifold with boundary, Nucl. Phys. B475 (1996) 94.

[5] E. Bergshoeff, E. Sezgin and P.K. Townsend, Supermembranes and 11 dimensional supergravity, Phys. Lett. B189 (1987) 75.

[6] E. Bergshoeff, E. Sezgin and P.K. Townsend, Properties of the elevendimensional supermembrane theory, Ann. Phys. (N.Y.) 185 (1988) 330.

[7] M.J. Duff, P.S. Howe, T. Inami and K.S. Stelle, Superstrings in D=10 from supermembranes in $D=11$, Phys. Lett. B191 (1987) 70.

[8] J. Polchinski, Dirichlet branes and Ramond-Ramond charges, Phys. Rev. Lett. 75 (1995) 184.

[9] E. Witten, Bound states of strings and p-branes, Nucl. Phys. B460 (1996) 335.

[10] S. de Alwis, A note on brane tension and M-theory, Phys. Lett. B388 (1996) 291; Anomaly cancellation in M-theory, Phys. Lett. B 392 (1997) 332.

[11] E. Dudas and J. Mourad On the strongly coupled heterotic string, hep-th 9701048, Phys. Lett. B (to appear).

[12] P.K. Townsend, D-branes from M-branes, Phys. Lett. B373 (1996) 68.

[13] A. Strominger, Open p-branes, Phys. Lett B383 (1996) 44.

[14] E. Bergshoeff, M. de Roo, M.B. Green, G. Papadopoulos and P.K. Townsend, Duality of type II 7-branes and 8-branes, Nucl. Phys. B470 (1996) 113. 
[15] P.S. Howe and E. Sezgin, Superbranes, Phys. Lett B390 (1997)133.

[16] G. Papadopoulos and P.K. Townsend, Kaluza-Klein on the brane, Phys. Lett. B393 (1997) 59.

[17] F. Aldabe, Heterotic and type I strings from twisted supermembranes, Nucl. Phys. B473 (1996) 63.

[18] K. Lechner and M. Tonin The cancellation of worldsheet anomalies in the D=10 Green-Schwarz heterotic string sigma-model, Nucl. Phys. B475 (1996) 535 .

[19] L. Alvarez-Gaume and E. Witten Gravitational anomalies, Nucl. Phys. B234 (1984) 269.

[20] E. Bergshoeff, E. Sezgin, and P.K. Townsend, Superstring actions in D=3,4,6,10 curved superspace, Phys. Lett. B169 (1986) 191.

[21] M. Tonin, Consistency condition for $k$-anomalies and superspace constraints in quantum heterotic superstrings, Int. J. Mod. Phys. A4 (1989) 1983.

[22] A. Candiello, K. Lechner and M. Tonin, k-anomalies and space-time supersymmetry in the Green-Schwarz heterotic superstring, Nucl. Phys. B438 (1995) 67.

[23] M.J. Duff, J.T. Liu, R. Minasian, Eleven dimensional origin of string/string duality: A One Loop Test, Nucl.Phys. B452 (1995) 261.

[24] E. Witten, Five-branes and M-Theory on an orbifold, Nucl.Phys. B463 (1996) 383-397.

[25] C. Vafa, E. Witten, A one-loop test of string duality, Nucl. Phys. B447 (1995) 261.

[26] E. Witten, On flux quantization in $M$-Theory and the effective action, hepth/9609122.

[27] I. Bandos, K. Lechner, A. Nurmagambetov, P. Pasti, D. Sorokin, M. Tonin, Covariant Action for the Super-Five-Brane of M-Theory, hep-th 9701149.

[28] M. Aganagic, J. Park, C. Popescu, J. H. Schwarz World-Volume Action of the $M$ Theory Five-Brane, hep-th 9701166. 